\title{
Pseudomonas-Related Vasculitis
}

National Cancer Institute

\section{Source}

National Cancer Institute. Pseudomonas-Related Vasculitis. NCI Thesaurus. Code C97118.

A necrotizing vasculitis caused by Pseudomonas aeruginosa. 